\title{
Application of ultrasound- assisted extraction method to recover betalains and polyphenols from red beetroot waste
}

\author{
Authors \\ Sumali N. Fernando $+\neq$, Kelly Wood $\neq$ Emmanouil Papaioannou\#, Lisa J. Marshall †, Natalia \\ Sergeevat, and Christine Boesch ${ }^{*} *$ \\ $\dagger$ School of Food Science and Nutrition, Faculty of Environment, University of Leeds, LS2 9JT, \\ United Kingdom. \\ $\ddagger$ School of Chemistry, Faculty of Engineering and Physical Sciences, University of Leeds, LS2 \\ 9JT, United Kingdom. \\ \#Department of Engineering, Faculty of Science and Technology, Lancaster University, LA1 \\ 4YW, Lancaster, United Kingdom.
}

Number of pages: 6

Number of figures: 5

Number of tables: 1 

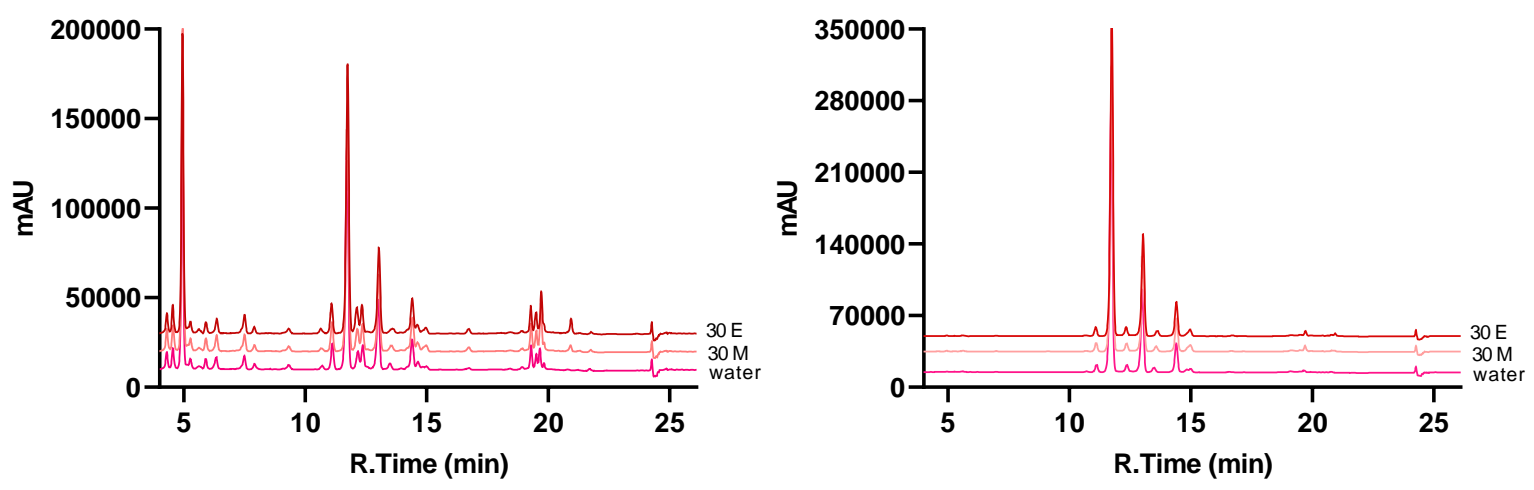

Figure S1. Betalain pattern of extracts. Presented are the different betalain compounds extracted with water, $30 \%$ methanol $(30 \mathrm{M})$ and $30 \%$ ethanol $(30 \mathrm{E})$. 
(A)

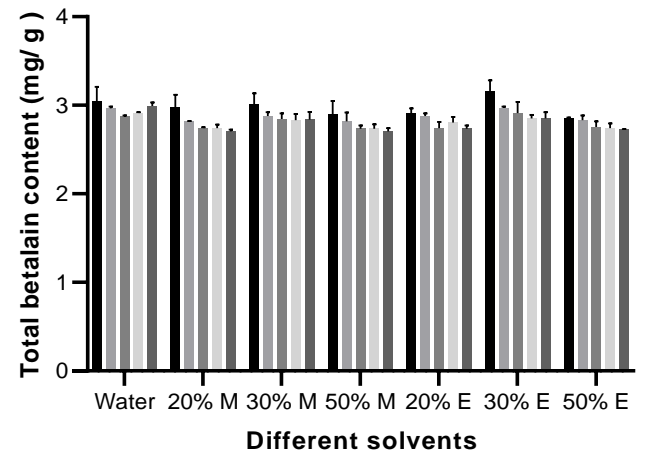

(C)

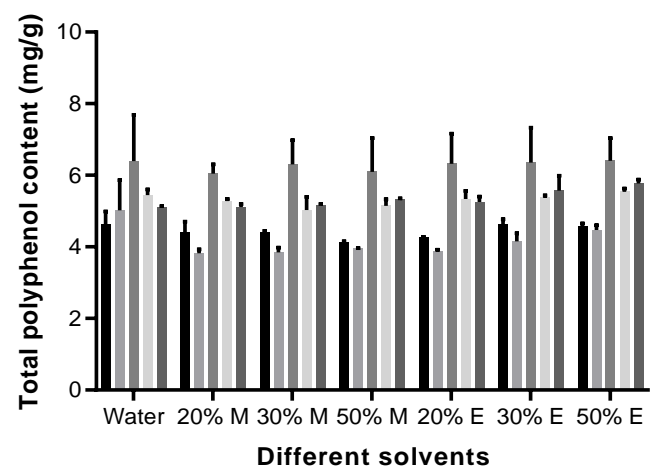

(E)

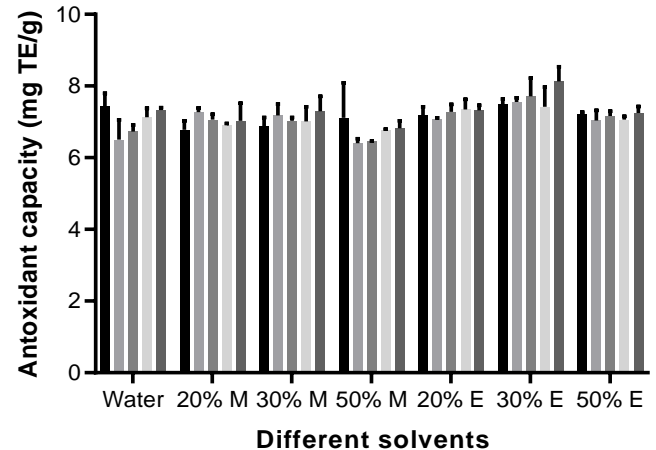

(B)

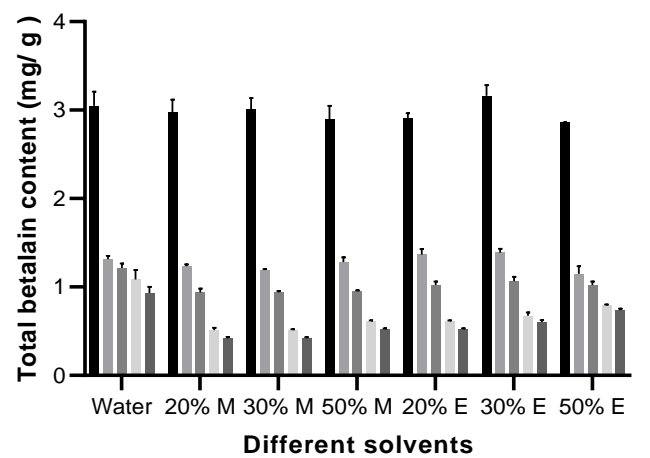

(D)

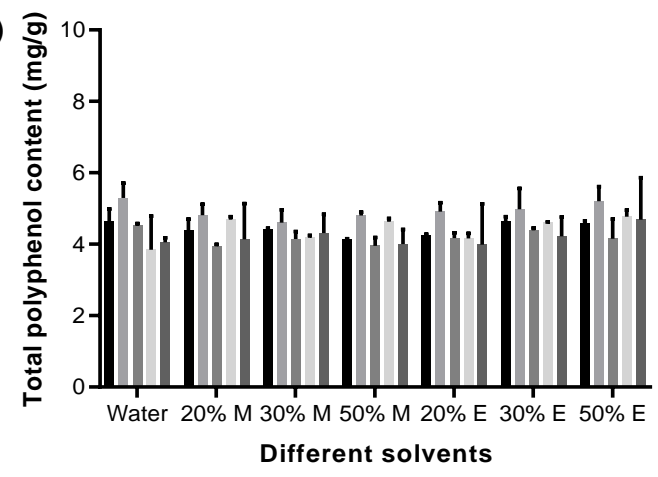

(F)

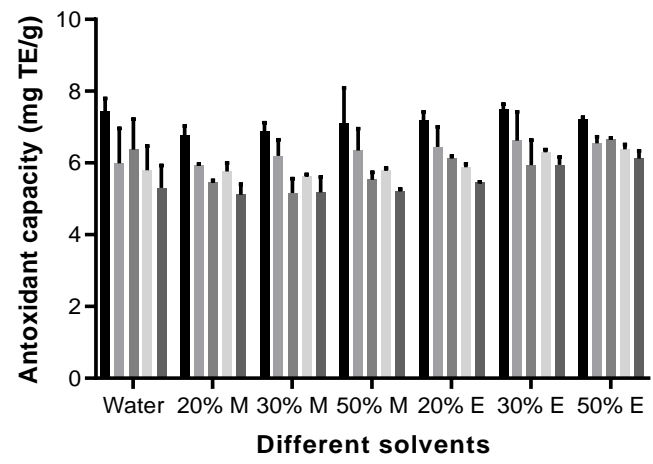

Figure S2. Variation of total betalain content, total polyphenol content and antioxidant activity (TEAC -Trolox equivalent antioxidant capacity) of the different extracts during a 4-week storage at $-20{ }^{\circ} \mathrm{C}(\mathrm{A}),(\mathrm{C}),(\mathrm{E})$ and room temperature (B), (D), (F). Data are mean with $\pm \mathrm{SD}(\mathrm{n}=2$, independent extractions). 
(A)

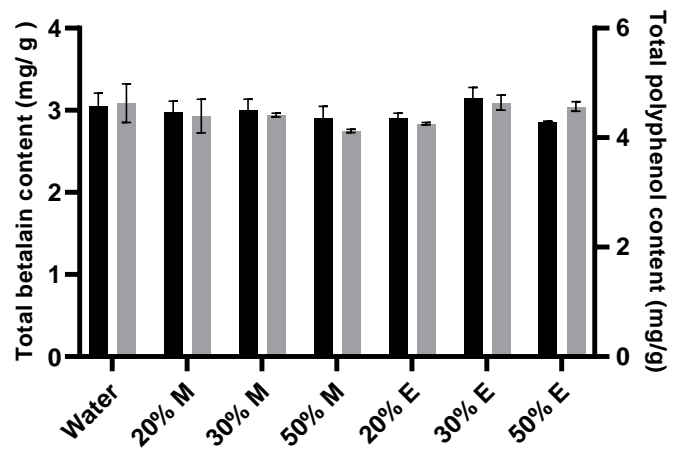

(B)

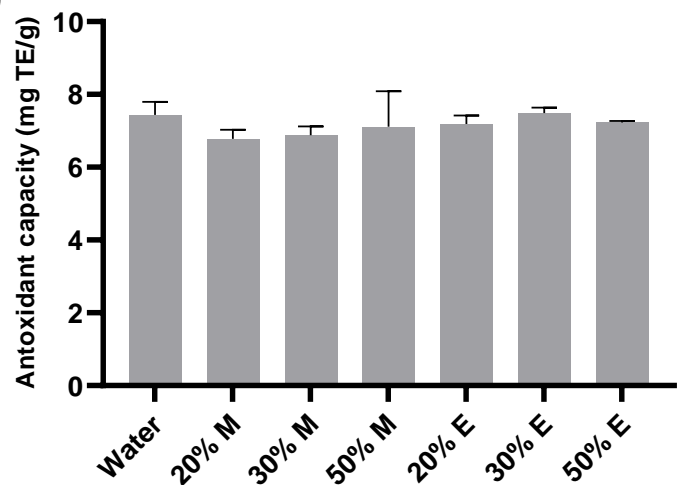

Figure S3. Effects of water, methanol and ethanol solvent mixtures on total betalain content, total polyphenol content and antioxidant activity in red beetroot extracts (initial values). Data are mean with \pm SD of three independent extractions.

Table S1. Pearson correlation coefficients (r) between colour values ( $\mathrm{L}, \mathrm{a}, \mathrm{b}, \mathrm{c}, \mathrm{h})$ and total betalain content

\begin{tabular}{crc}
\hline & \multicolumn{1}{c}{$\begin{array}{l}\text { Total } \\
\text { Colour trait }\end{array}$} & $\begin{array}{c}\text { betalain } \\
\text { r content }\end{array}$ \\
\cline { 2 - 3 } & -0.9074 & $<0.0001$ \\
\hline $\mathrm{L}^{*}$ & 0.8967 & $<0.0001$ \\
$\mathrm{a}^{*}$ & -0.8807 & $<0.0001$ \\
$\mathrm{~b}^{*}$ & 0.5930 & $<0.0001$ \\
$\mathrm{c}^{*}$ & -0.9256 & $<0.0001$ \\
$\mathrm{~h}$ & 0.9820 & $<0.0001$ \\
$\mathrm{Lab}$ & 0.9840 & $<0.0001$ \\
$\mathrm{a} / \mathrm{b}$ &
\end{tabular}

$\mathrm{L}^{*}=$ lightness or darkness; $\mathrm{a}^{*}=$ redness or greenness; $\mathrm{b}^{*}=$ yellowness or blueness; $\mathrm{c}^{*}=$ chroma; $\mathrm{h}=$ hue angle, $\mathrm{a} / \mathrm{b}-$ ratio that indicate the quantitative development of colour 


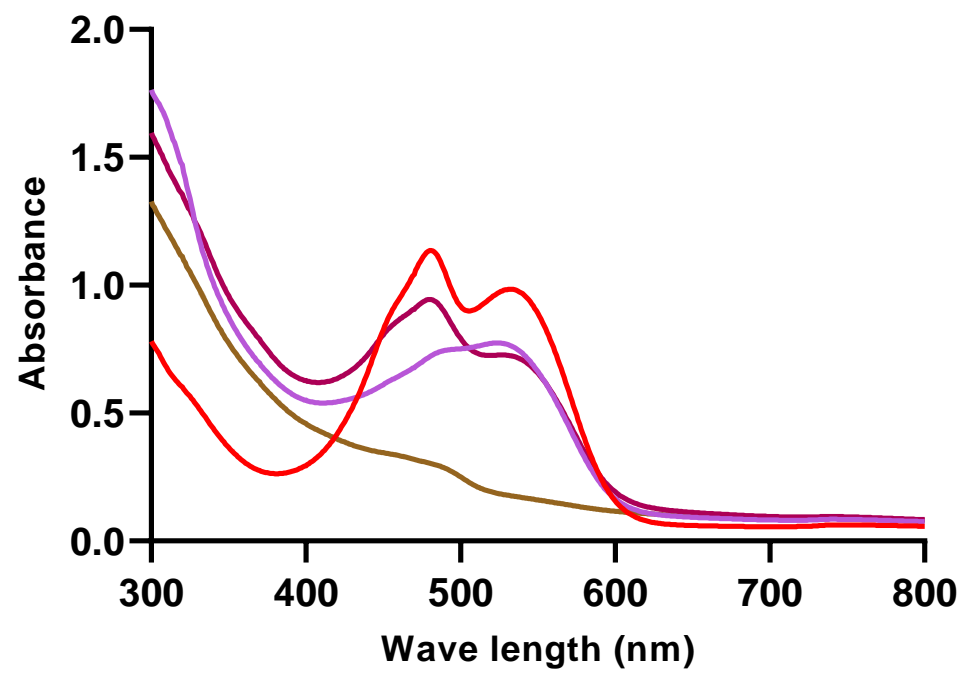

— Beetroot powder — Beetroot juice - Beetroot waste (FD) - Beetroot waste (AD)

Figure S4. UV-vis scan of beetroot samples showing the characteristics at $536 \mathrm{~nm}$ and $486 \mathrm{~nm}$ wavelength. 

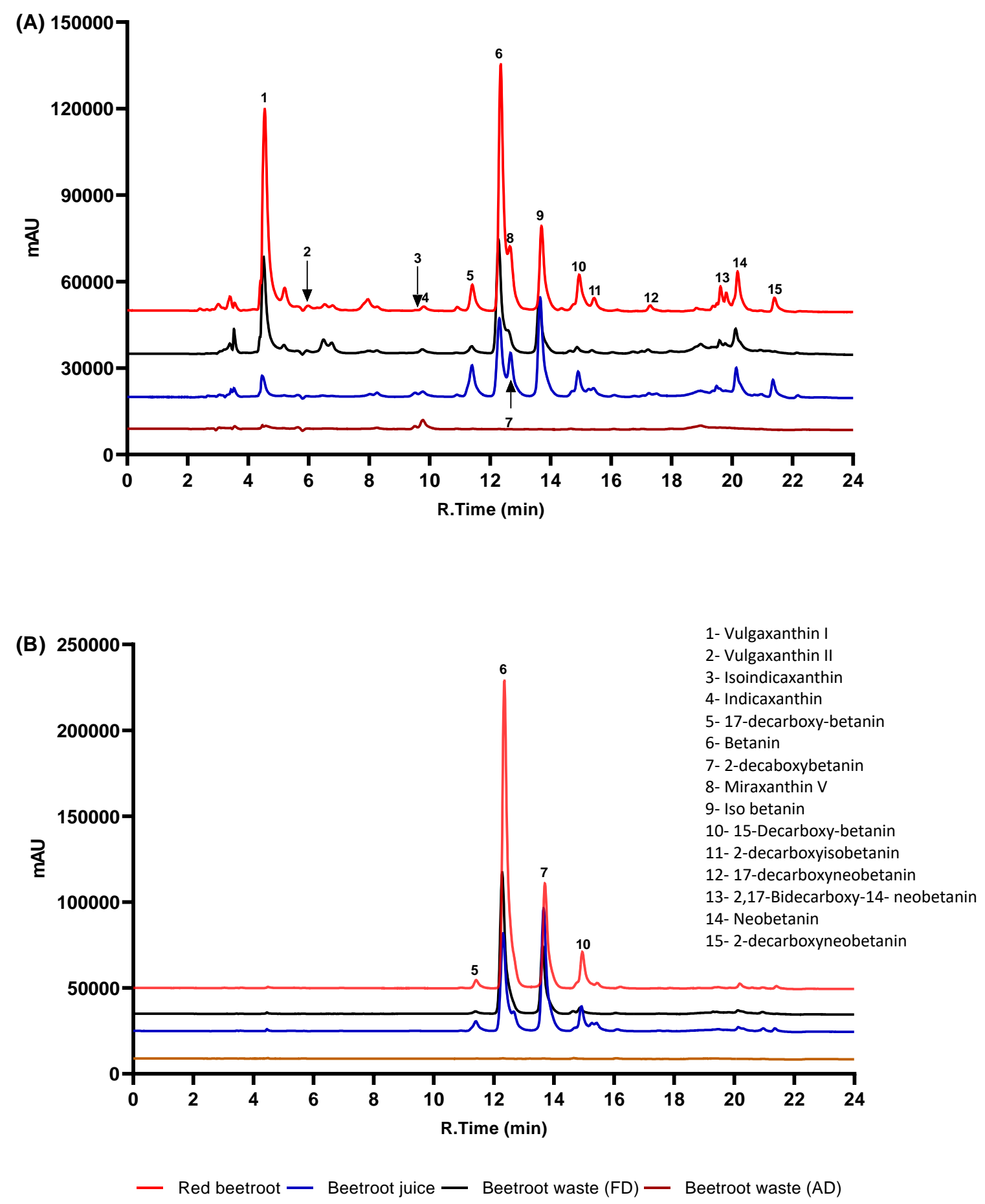

Figure S5. HPLC chromatograms of different red beetroot samples. Elution monitored at $486 \mathrm{~nm}$ (betaxanthins, A) and $536 \mathrm{~nm}$ (betcayanins, B). ESI-MS positive ion mode was used to identify the peaks. 